\title{
Post-ictal missing dentures
}

\author{
Edward D. Foley, MD 1 - Zhuo Sun, MD • Manuel R. Castresana, MD, FCCM, FACA, FASA
}

Received: 2 August 2017/Revised: 9 August 2017/Accepted: 14 August 2017/Published online: 17 August 2017

(C) Canadian Anesthesiologists' Society 2017

A 40-yr-old epileptic woman presented to the hospital following a witnessed seizure; her partial lower denture could not be found. On evaluation, the patient had copious oral secretions, was drooling, and had a weak voice. A lateral neck plain radiograph (Figure) showed a transversely oriented wire structure anterior to the C6-7 vertebrae and posterior to the cricoid cartilage along with air in the retropharyngeal and prevertebral tissues, suggesting upper esophageal perforation. Fibreoptic nasotracheal intubation was performed followed by a computed tomography (CT) scan that confirmed posterior pharyngeal wall puncture and extensive air in the retropharyngeal space. Prophylactic vancomycin and piperacillin/tazobactam were administered. The foreign body was retrieved under general anesthesia from the right lateral postcricoid space in the proximal cervical esophagus.

The reported incidence of an esophageal location for impacted dentures varies from $0.4 \%$ to $17.6 \%$. $^{1}$ Although plain radiographs may well not always identify a swallowed denture, an $x$-ray investigation is recommended to exclude pneumomediastinum. Preferably, however, a CT scan should be performed. A barium contrast study is seldom helpful because it can coat all sides of a radiolucent object

E. D. Foley, MD $(\bowtie) \cdot$ Z. Sun, MD .

M. R. Castresana, MD, FCCM, FACA, FASA

Department of Anesthesiology and Perioperative Medicine,

Medical College of Georgia, Augusta University, Augusta, GA,

USA

e-mail: efoley@augusta.edu and make subsequent endoscopy more difficult. ${ }^{2}$ Early diagnosis and treatment reduce the mucosal inflammatory reaction and risk of infection. Uncomplicated cases of impacted dentures in the upper gastrointestinal tract can be managed with flexible or rigid endoscopic removal. Endoscopy failure or a complicated case - with gastrointestinal tract perforation, hemorrhage, or infection -usually requires surgical management. A thorough history and airway examination is important in patients with a diagnosis of denture ingestion. Airway examination, as part of the perioperative anesthesiology and post-ictal evaluation,

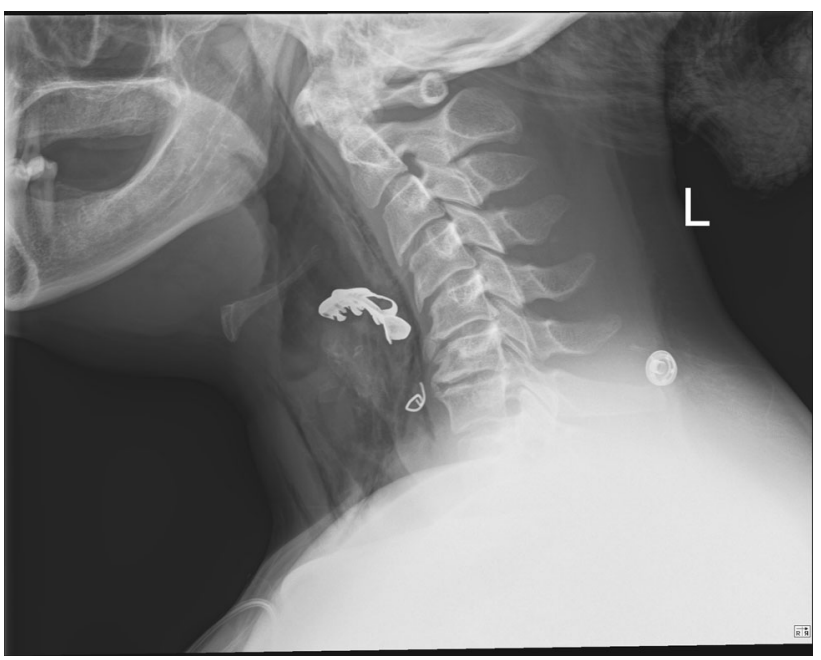

Figure Plain radiography of the lateral neck soft tissue shows the denture anterior to the C6-7 vertebrae and posterior to the cricoid cartilage. Air in the retropharyngeal/prevertebral tissues suggests upper esophageal perforation 
is especially important for edentulous patients with altered mental status and loss of consciousness.

Conflicts of interest None declared.

Editorial responsibility This submission was handled by Dr. Hilary P. Grocott, Editor-in-Chief, Canadian Journal of Anesthesia.

\section{References}

1. Gachabayov M, Isaev M, Orujova L, Isaev E, Yaskin E, Neronov $D$. Swallowed dentures: two cases and a review. Ann Med Surg (Lond) 2015; 4: 407-13.

2. Hashmi $S$, Walter J, Smith W, Latis $S$. Swallowed partial dentures. J R Soc Med 2004; 97: 72-5.

Financial support None. 